\title{
A Delay and Energy Aware Techique for Wireless Sensor Networks using Regular Sleep and Wake Periods
}

\author{
Balaji M. Kore \\ M.E (CN) \\ Department of Computer Engineering, SKNCOE, Pune.
}

\author{
S.K. Pathan \\ Professor \\ Department of Computer Engineering, SKNCOE, Pune.
}

\begin{abstract}
Wireless sensor network is collection of large numbers of wireless sensor nodes to collect information from their sensing terrain. Wireless sensor nodes are battery-powered devices energy saving is always important to the lifetime of wireless sensor network. Recently many algorithms are available to track energy saving problems in wireless sensor network to increase life time of network the algorithms are top-down approach and bottom-up approach but there are problems with this techniques. The proposed technique is a delay aware technique for wireless sensor network using regular sleep and wake periods. The objective of proposed network structure is to minimize delay and to increase lifetime of wireless sensor network. The proposed network formation technique is sleep and wake period with top-down approach.
\end{abstract}

\section{General Terms:}

Wireless Sensor Network, Energy Utilization Of Wireless Sensor Network

\section{Keywords:}

Top-Down approach, Bottom-Up approach, Sleep and Wake Periods.

\section{INTRODUCTION}

Strong adaptability, comprehensive sensing coverage, and high fault tolerance are some of the unique advantages of wireless sensor networks. Wireless sensor networks consist of large amounts of wireless sensor nodes, which are compact, lightweighted, and battery-powered devices that can be used in virtually any environment. Because of these special characteristics, sensor nodes are usually deployed near the targets of interest in order to do close-range sensing. The data collected will undergo in-network processes and then return to the user who is usually located in a remote site. Most of the time, wireless sensor nodes are located in extreme environments, where are too hostile for maintenance. Sensor nodes must conserve their scarce energy by all means and stay active in order to maintain the required sensing coverage of the environment.[1]

Generally clustering is used to save the energy of the node. A network with clustering is collection of many clusters. Within each cluster there is cluster head $(\mathrm{CH})$ and cluster member $(\mathrm{CM})$.The cluster head is responsible to collect data from cluster member directly or in multihop manner. By using this the number of node involved in transmission is redused therefour required energy is also minimum. The cluster head collect data from cluster mem-

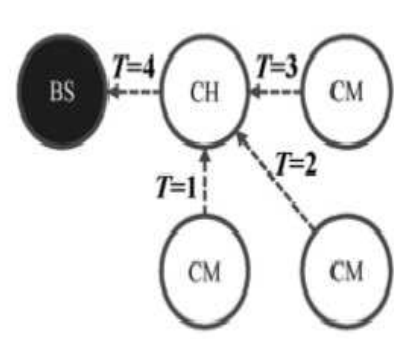

(a)

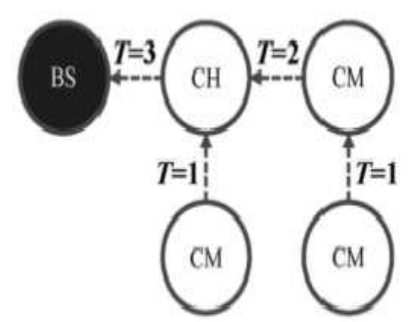

(b)
Fig. 1. (a)Data collection in two-hop network and (b)Data collection in multihop network

ber. The data generated by cluster member(node) is highly corelated thus it is capable to fusing all received packets into a single packet by means of data/decision fusing technique.

The aim of this paper is to minimize delay and increase the energy of node by using sleep and wake periods with top-down approach.The proposed algorithm works between data link layer and network layer.

\section{RELATED WORK}

In wireless sensor network maximum energy is utilized in wireless communication.The energy utilized is directly propotional to corresponding distance. The long distance communication of node with base station is not good idea. One way to reduce energy is by using clustering algorithm. The Clustering algorithm works as follows

1)Organization of nodes into cluster. 2)With the cluster one node elected as cluster head and other are cluster member. 3)The cluster head collect data from cluster member, combine packet by using data/decision fusion technique. Submit fused data to remote base station.

Here cluster head only participate in long transmission therefour energy of other node is redused.

Research has been conducted on redusing energy by forming cluster with appropriate network structure.Heinzelman et al. proposed a clustering algorithm called LEACH Low-Energy Adaptive Clustering Hierarchy [1].The LEACH protocol is a hierarchical protocol in which most nodes transmit to cluster heads. The operation of the LEACH protocol consists of two phases:

1)The Setup phase. In setup phase the cluster heads are selected. The cluster head agrregate, compress, forword data packet to 
the base station. Each node determines whether it will become a cluster head, in this round, by using a stochastic algorithm at each round. If a node becomes a cluster head for one time, it cannot become cluster head again for $\mathrm{q}$ rounds, where $\mathrm{q}$ is the desired percentage of cluster heads. Thereafter, the probability of a node to become acluster head in each round is 1/q. This rotation of cluster heads leads to a balanced energy consumption to all the nodes and hence to a longer lifetime of the network.2)The Steady State Phase. In the Steady State Phase, the data is sent to the base station.The duration of the steady state phase is longer than the duration of the setup phase in order to minimize overhead. Moreover, each node that is not a cluster head selects the closest cluster head and joins that cluster. After that the cluster head creates a schedule for each node in its cluster to transmit its data. The main advantage of LEACH is that it outperforms conventional communication protocols, in terms of energy dissipation, ease of configuration, and system lifetime/quality of the network. Providing such a low energy, wireless distributed protocol will help pave the way in a WSN. How ever, LEACH uses single-hop routing where each node can transmit directly to the cluster-head and the sink. Therefore, it is not recommended for networks that are deployed in large regions. Furthermore, the dynamic clustering may results to extra overhead, e.g. head changes, advertisements etc., which may diminish the gain in energy consumption.[2]

Low-Energy Adaptive Clustering Hierarchy Centralized (LEACH-C): The LEACH-C utilizes the base station for cluster formation, unlike LEACH where nodes self-configure themselves into clusters.Initially in the LEACH-C, the Base Station (BS) receives information regarding the location and energy level of each node in the network. After that, using this information, the BS finds a predetermined number of cluster heads and configures the network into clusters. The cluster groupings are chosen to minimize the energy required for non-cluster-head nodes to transmit their data to their respective cluster heads. The improvements of this algorithm compared to LEACH are the following: 1)The BS utilizes its global knowledge of the network to produce clusters that require less energy for data transmission. 2)Unlike LEACH where the number of cluster heads varies from round to round due to the lack of global coordina- tion among nodes, in LEACH-C the number of cluster heads in each round equals a predetermined optimal value.[2] Threshold sensitive Energy Efficient sensor Network protocol (TEEN): The TEEN is a hierarchical protocol designed for the conditions like sudden changes in the sensed attributes such as temperature.The responsiveness is important fortime-critical applications, in which the network is operated in a reactive mode. The sensor network architecture in TEEN is based on a hierarchical grouping where closer nodes form clusters and this process goes on the second level until the sink is reached. In this scheme the cluster-head broadcasts to its members the Hard Threshold (HT) and the Soft Threshold (ST). The HT is a threshold value for the sensed attribute. It is the absolute value of the attribute beyond which, the node sensing this value must switch on its transmitter and report to its cluster head. The ST is a small change in the value of the sensed attribute which triggers the node to switch on its transmitter and transmit. The nodes sense their environment continuously. The first time a parameter from the attribute set reaches its hard threshold value, the node switches on its transmitter and sends the sensed data. The sensed value is stored in an internal variable in the node, called the sensed value (SV). The main advantage of TEEN is that it works well in the conditions like sudden changes in the sensed attributes such as temperature. On the other hand, in large area networks and when the number of layers in the hierarchy is small, TEEN tends to consume a lot of energy, because of long distance transmissions. Moreover, when the number of layers increases, the transmissions become shorter and overhead in the setup phase as well as the operation of the network exist.[2]
Lindsey and Raghavendra proposed another clustering algorithm called PEGASIS Power-Efficient Gathering in Sensor Information Systems. The PEGASIS protocol is chain based protocol and improvement in LEACH protocol. In PEGASIS each node communicate only with a nearby neighbour to send and receive packet. It takes turns transmitting to the base station,thus redusing the amount of energy spent per round. By using greedy algorithm we can form a chain considering starting node randomly or base station. By minimizing the number of cluster heads, the energy consumed in long distance transmission is further minimized.In general, the PEGASIS protocol presents twice or more performance in comparison with the LEACH protocol However, the PEGASIS protocol causes the redundant data transmission since one of the nodes on the chain has been selected. Unlike LEACH, the transmitting distance for most of the nodes is reduced in PEGASIS. Experimental results show that PEGASIS provides improvement by factor 2 compared to LEACH protocol for $50 \mathrm{~m}$ x $50 \mathrm{~m}$ network and improvement by factor 3 for $100 \mathrm{~m} \times 100 \mathrm{~m}$ network. The PEGASIS protocol, however, has a critical problem that is the redundant transmission of the data. The cause of this problem is that there is no consideration of the base stations location about the energy of nodes when one of nodes is selected as the head node.[2]

Tan and Korpeoglu developed PEDAP, which is based on the idea of a minimum spanning tree (MST). Besides minimizing the amount of long distance transmission, the communication distances among sensor nodes are minimized[1]. Fonseca et al. proposed the collection tree protocol (CTP). The CTP is a kind of gradient-based routing protocol which uses expected transmissions (ETX) as its routing gradient. ETX is the number of expected transmissions of a packet necessary for it to be received without error. Paths with low ETX are expected to have high throughput. Nodes in a network using CTP will always pick a route with the lowest ETX. In general, the ETX of a path is proportional to the corresponding path length. Thus, CTP can greatly reduce the communication distances among sensor nodes[1].Another important approach is Top-Down approach.In Top-Down approach all nodes participate in transmission by using this delay can be minimized. The overall approach is going to execute at base station. Base station is responsible for all the activities. The another approach is Bottom-Up aprroach. In Bottom-Up approach join the cluster of the same size togrther. It is scalable than Top-Down approach. In Bottom-Up approach the base station is selected from among nodes depending on energy of node.

\section{PROGRAMMER'S DESIGN}

Wireless Sensor network contain large number of wireless sensor nodes to collect information from other nodes. Wireless sensor nodes are battery powered devices energy saving is always important to the lifetime of wireless sensor network. Here we are going to organize the wireless nodes in such way that the delay is minimum and energy of node is consumed less by using different algorithm to organize the nodes.The algorithm we are going to use Top-Down approach using regular sleep and wake periods.

\subsection{Mathematical Model}

A wireless sensor node can be made of using three major units,namely the microcontroller unit(MCU), the transceiver unit(TCR), and the sensor board(SB). Each of which consume a certain amount of energy while operating. The energy consumed by wireless sensor node $\mathrm{j}$ can be given as

$$
E_{j_{-} S N}=E_{j_{-} M C U}+E_{j_{-} T C R}+E_{j_{-} S B}
$$

where $E_{j_{-} M C U}$ denotes energy consumed by microcontrol unit, $E_{j_{-} T C R}$ denotes energy consumed by transreciver and $E_{j_{-} S B}$ 
denotes energy by sensor board.The $E_{j_{-} T C R}$ can be further classified as

$$
E_{j_{-} T C R}=E_{j_{-} T C R_{-} R X}+E_{j_{-} T C R_{-} T X}\left(d_{j}\right)
$$

Here $E_{j_{-} T C R_{-} R X}$ represents energy consumed by transreciver in receiving mode, while $E_{j_{-} T C R_{-} T X}\left(d_{j}\right)$ represents energy consumed by the transreceiver to transmit for distance of $d_{j}$ . The Total energy consumed by a network of $\mathrm{N}$ nodes is given by

$$
\begin{array}{r}
E_{T O T}(N)=(3) \\
\sum_{j=1}^{N} E_{j_{-} M C U}+E_{j_{-} T C R \_R X}+E_{j_{-} T C R \_T X}\left(d_{j}\right)+E_{j_{-} S B}
\end{array}
$$

Normally $E_{j_{-} M C U}, E_{j_{-} T C R}, E_{j_{-} T C R_{-} R X}$ are constant. But $E_{j_{-} T C R_{-} T X}\left(d_{j}\right)$ is function of $d_{j}$ which is depends on network structure. Therefour (3) can be modified as

$$
E_{T O T}(N)=C_{1}+\sum_{j=1}^{N} E_{j \_T C R \_T X}\left(d_{j}\right)
$$

Here $C_{1}$ is constant. The $E_{j_{-} T C R_{-} T X}\left(d_{j}\right)$ can be further expressed as

$$
E_{j_{-} T C R_{-} T X}\left(d_{j}\right)=E_{j_{-} T C R_{-} E C}+E_{j_{-} T C R_{-} P A d_{j}^{2}}
$$

where $E_{j_{-} T C R_{-} E C}$ is the energy utilized by TCR's electronic circuitry, while $E_{j_{-} T C R_{-} P A}$ is the energy consumed by power amlifier of TCR both are constant therefour (4) can be expressed as

$$
E_{T O T}=C_{1}+C_{2}+C_{3} \sum_{j=1}^{N} d_{j}^{2}
$$

Here $C_{1}$ and $C_{2}$ are constant Here (6) shows that total energy utilized of network can be minimized by reducing distance. To organize node with minimum distance the proposed algorithm is Top-Down approach with perodic sleep and wake periods.

\subsection{Top-Down Approach with perodic sleep and wake periods}

In this approach, the base station is assumed to have the coordinates of all sensor nodes in the network. The algorithm is going to execute at base station. At the last base station will inform to all nodes make a data connection from the appropriate network structure. To construct the network for $N=2^{0}$ and $N=2^{1}$ is simple. For networks with $N=2^{p}$ nodes, $\mathrm{p}=2,3, .$. the algorithm is shown below

1)The algorithm starts with considering the whole network as a fully connected network. In this paper, the term connected refers to the existence of a data link between two wireless sensor nodes which is used to transmit data packets in the data collection processes. Two wireless sensor nodes are defined as disconnected from each other if there does not exist any direct data link between them. The connection degree of a wireless sensor node is telling the number of data links associated with such node. A node with connection degree of 3 implies that such a node has formed three data links with three other nodes.For a network of $N=2^{p}$ nodes where $\mathrm{p}=2,3, .$. each node will begin with degree equal to $N-1$. The nodes will form the set $G_{s}=1$ set $k=N / 2$

2)Select k nodes from set $H_{s}=1$ to form set $H_{s}+1$, such that $d_{i, j}^{2}$ is maximized.Here $d_{i, j}$ represent geographical distance between nodes $\mathrm{i}$ and $\mathrm{j}$. The rest of the nodes from $H_{s}=1$ will from set $H_{s}+1$. The algorithm will then remove all connections among nodes within $G_{s}+1$. Set iterations $\mathrm{s}=\mathrm{s}+1$ and b=b/2. 3)Repeat step 2 until $k<2$ set $\mathrm{r}=2$
4)Nodes with degree $\mathrm{N}$-r form set L. Nodes with degree greater than N-r form set $\mathrm{U}$ such that set $\mathrm{L}$ and set $\mathrm{U}$ are of the same number of nodes. Each node in set L is only connected to a single node in set $\mathrm{U}$. Set $\mathrm{r}=\mathrm{r} * 2$

5)Repeat step 4 until $\mathrm{r}=\mathrm{N}$.

\section{RESULTS AND DISCUSSION}

Top-Down approach have some drawback's as follows All nodes are participating in the transmission by using this delay can be minimized but network lifetime cannot improved to improve this we can use sleep and wake period with top-down approach by using this minimum node going to participate in the data transfer so lifetime of network is improved. Steps in Sleep And Wake Period With Top-Down Approach:

1)Initially All nodes are not participate in transmission. Only few nodes are wake-up initially.

2)After some time sleep node becomes activate and take participate in the transmission.

3)When the new nodes are added the path to communicate to base station is calculated once again.

4)Likewise the working sleep and wake period with top-down approach take place.

\section{CONCLUSION}

In this paper, a delay-aware data collection network structure and formation algorithm are proposed. The proposed network formation algorithm is top-down approach with sleep and wake periods. The performance of proposed network algorithm is compared with top-down approach. The proposed network structure minimized delay in communication and increade lifetime of network. The proposed network structure can highly reduce the data collection time while keeping the total communication distance and the network lifetime at minimum values.

\section{REFERENCES}

[1] C.M.Lau Chi-Tsun Cheng, Chi K. Tse "A delay-aware data collection network structure for wireless sensor networks".IEEE SENSORS JOURNAL,VOL. 11, NO. 3, MARCH 2011

[2] Nikolaos A. Pantazis, Stefanos A. Nikolidakis and Dimitrios D. Vergados, Senior Member,IEEE "Energy-Efficient Routing Protocols in Wireless Sensor Networks:A Survey".IEEE COMMUNICATIONS SURVEYS TUTORIALS, ACCEPTED FOR PUBLICATION,2012 IEEE

[3] W. B. Heinzelman, A. P. Chandrakasan, and H. Balakrishnan, An ap- plication-specific protocol architecture for wireless microsensor net- works, IEEE Trans. Wireless Commun., vol. 1, no. 4, pp. 660670, Oct. 2002.

[4] S. Lindsey and C. S. Raghavendra, PEGASIS: Powerefficient gath- ering in sensor information systems, in Proc. IEEE Conf. Aerosp., Big Sky, MT, USA, Mar. 2002, vol. 3, pp. 11251130.

[5] H. . Tan and . Krpeoglu, Power efficient data gathering and aggre- gation in wireless sensor networks, ACM SIGMOD Record, vol. 32, no. 4, pp. 6671, Dec. 2003.

[6] R. Fonseca, O. Gnawali, K. Jamieson, S. Kim, P. Levis, and A. Woo, The collection tree protocol, TinyOS Enhancement Proposals (TEP), vol. 123, Dec. 2007.

[7] D. S. J. D. Couto, High-Throughput routing for multihop wireless networks, Ph.D. dissertation, Dept. Elect. Eng. Comput. Sci., Massa- chusetts Inst. Technol., Cambridge, MA, 2004. 
[8] O. Tekdas, J. H. Lim, A. Terzis, and V. Isler, Using mobile robots to harvest data from sensor fields, IEEE Wireless Commun. Mag., vol. 16, no. 1, pp. 2228, Feb. 2009.

[9] A. Manjeshwar and D. P. Agrawal, TEEN: A routing protocol for enhanced efficiency in wireless sensor networks, in Proc. 15th Int. Symp. Parallel Distrib. Process., (IPDPS 2001), San Francisco, CA, Apr. 2001, pp. 20092015.
[10] A. Manjeshwar and D. P. Agrawal, APTEEN: A hybrid protocol for efficient routing and comprehensive information retrieval in wireless sensor networks, in Proc. 16th Int. Symp. Parallel Distrib. Process., (IPDPS 2002), Fort Lauderdale, FL, Apr. 2002, pp. 195202. 